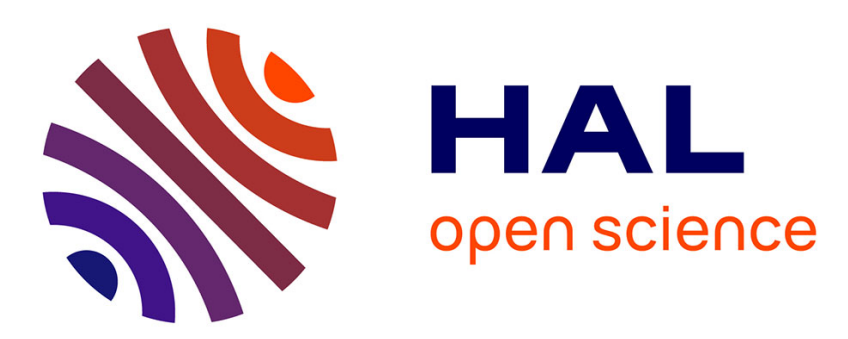

\title{
A design solution to the problem of adaptive output regulation for nonlinear minimum-phase systems
}

\author{
Alberto Isidori, Lorenzo Marconi, Laurent Praly
}

\section{To cite this version:}

Alberto Isidori, Lorenzo Marconi, Laurent Praly. A design solution to the problem of adaptive output regulation for nonlinear minimum-phase systems. 49th IEEE Conference on Decision and Control, Dec 2010, Atlanta, United States. pp.2704-2709, 10.1109/CDC.2010.5717217 . hal-00554882

HAL Id: hal-00554882

https://hal-mines-paristech.archives-ouvertes.fr/hal-00554882

Submitted on 11 Jan 2011

HAL is a multi-disciplinary open access archive for the deposit and dissemination of scientific research documents, whether they are published or not. The documents may come from teaching and research institutions in France or abroad, or from public or private research centers.
L'archive ouverte pluridisciplinaire HAL, est destinée au dépôt et à la diffusion de documents scientifiques de niveau recherche, publiés ou non, émanant des établissements d'enseignement et de recherche français ou étrangers, des laboratoires publics ou privés. 


\title{
A design solution to the problem of adaptive output regulation for nonlinear minimum-phase systems
}

\author{
A. Isidori, L. Marconi, L. Praly
}

\begin{abstract}
We propose a solution to the problem of adaptive output regulation for nonlinear minimum-phase systems that does not rely upon conventional adaptation schemes to estimate the frequency of the exogenous signals. The proposed approach relies upon regression tools to derive a nonlinear internal model able to offset the presence of an unknown number of harmonics of uncertain amplitude, phase and frequency. The design methodology guarantees asymptotic regulation in the case the dimension of the regulator is sufficiently large in relation to the effective number of harmonics acting on the system. On the other hand, in the case of under-dimensioned internal model, a bounded steady-state regulation error is ensured whose amplitude, though, can be arbitrarily decreased by acting on a design parameter of the regulator. The proposed tool is also shown to be effective to deal with the larger class of nonlinear but linearly parameterized uncertain exosystems.
\end{abstract}

\section{INTRODUCTION}

This work focuses on the problem of output regulation for minimum-phase nonlinear systems in case the exogenous signal is given by a set of uncertain harmonic oscillators, namely the problem usually referred to as adaptive output regulation. Since the original work [11] where the problem of adaptive output regulation was formulated and a design solution provided, a number of contributions have been given on the subject both for linear and nonlinear systems. Most of the works proposed in this context adopt a variety of tools and ideas typical of the adaptive literature in order to estimate the exosystem uncertainties or, more in the spirit of the internal model principle, the control law needed to fulfill the regulation objective. In [3] the theory of adaptive observers for nonlinear systems was shown to be effective in designing nonlinear internal models in a semiglobal minimum-phase setting. In [9] and [4] global adaptive tools was applied to solve the problem for linear and nonlinear systems in a global setting, with the case of "large-scale systems" dealt with in [13]. An application of adaptive output regulation was proposed in [1] regarding an implicit fault tolerance problem for induction machines. In [10] an adaptive solution to the problem of compensating for the effect of measurement noise with uncertain frequencies was formulated in terms of output regulation and solved by using an "hybrid" control strategy. In that paper it was emphasized how persistence of excitation conditions, crucial in the adaptive literature for parameter estimation, are not

A. Isidori is with DIS, Università di Roma "La Sapienza", Rome, Italy albisidori@dis.uniroma1.it.

L. Marconi is with C.A.SY. - DEIS, University of Bologna, Bologna, Italy lorenzo.marconi@unibo.it.

L. Praly is with École des Mines de Paris, Fontainebleau, France Laurent.Praly@mines-paristech.fr. indeed necessary in the problem of output regulation, in which the problem is to steer a regulation error to zero rather then estimating uncertain parameters. This fact is also implicit in [8] dealing with a more general class of regulation problems.

In this paper we add a further design methodology to the already rich scenario of adaptive output regulation. The proposed approach relies upon the theory of nonlinear highgain observers, proved to be effective in [2] within the context of nonlinear output regulation, in order to design a nonlinear internal model which does not rely upon an "explicit" adaptation law. The latter feature makes the problem at hand different from existing solutions by thus enriching the available design tools. We develop the theory in a general framework comprising the case of over- and underdimensioned internal models. In the first scenario, capturing the case in which the number of effective exogenous harmonics is over-estimated, we show that asymptotic regulation is achieved, without necessarily relying upon persistence of excitation. On the other hand, in the case the number of effective exogenous harmonics is under-estimated, the proposed controller ensures a bounded steady-state regulation error whose amplitude, though, can be arbitrarily decreased by acting on a design parameter of the regulator. The latter feature is interesting and, to best knowledge of the authors, never addressed in the related literature. At the end of the work we also sketch how the proposed methodology can be effective for the larger class of nonlinear but linearly parametrized exosystems. Further details in this direction will be presented in an extended journal version of this work which is under preparation.

\section{PROBLEM FORMULATION AND PRELIMINARIES}

We consider the smooth system

$$
\begin{aligned}
\dot{\mathbf{w}} & =s(\mathbf{w}) \\
\dot{z} & =f(\mathbf{w}, z, e) \\
\dot{e} & =q(\mathbf{w}, z, e)+u
\end{aligned}
$$

in which $u$ is the control input, $(z, e) \in \mathbb{R}^{n} \times \mathbb{R}$ the state with $e$ the regulation error, and with the initial conditions that are supposed to range in a known compact set $Z \times E \subset \mathbb{R}^{n} \times \mathbb{R}$. The variable $\mathbf{w} \in \mathbb{R}^{s}$ is an exogenous variable, modelling reference to be tracked and/or disturbances to be rejected, that is supposed to range on a compact set $\mathbf{W} \subset \mathbb{R}^{s}$ invariant for the exosystem dynamics $\dot{\mathbf{w}}=s(\mathbf{w})$.

In this setting we address the problem of nonlinear output regulation which consists of designing a error-feedback 
controller of the form

$$
\dot{\xi}=\alpha(\xi, e) \quad u=\beta(\xi, e)
$$

with initial condition in a compact set $\Xi \subset \mathbb{R}^{d}$, in such a way the trajectories of the resulting closed-loop system originating from $\mathbf{W} \times Z \times E \times \Xi$ are bounded and $\lim _{t \rightarrow \infty} e(t)=0$.

We approach the problem under customary assumptions in most of the contributions about output regulation. First, we suppose there exists a map $\pi: \mathbf{W} \rightarrow \mathbb{R}^{n}$ solution of the regulator equations

$$
\frac{d \pi(\mathbf{w})}{d \mathbf{w}} s(\mathbf{w})=f(\mathbf{w}, \pi(\mathbf{w}), 0) .
$$

Furthermore, we formulate a minimum-phase assumption that, in this context, reads as follow.

Assumption 1. The set

$$
\operatorname{graph}(\pi)=\left\{(\mathbf{w}, z) \in \mathbf{W} \times \mathbb{R}^{n}: z=\pi(\mathbf{w})\right\}
$$

is locally asymptotically stable for the system

$$
\dot{\mathbf{w}}=s(\mathbf{w}) \quad \dot{z}=f(\mathbf{w}, z, 0)
$$

with a domain of attraction of the form $\mathbf{W} \times \mathcal{D}$ where $\mathcal{D}$ is an open set satisfying $\mathcal{D} \supset Z$. $\triangleleft$

In the forthcoming discussion we shall denote by $u^{\star}(\mathbf{w})$ the following

$$
u^{\star}(\mathbf{w}):=-q(\mathbf{w}, \pi(\mathbf{w}), 0) .
$$

For the previous class of systems it is known that the problem in hand can be solved if one is able to design a locally Lipschitz function $F: \mathbb{R}^{d} \rightarrow \mathbb{R}^{d}$, a continuous function $\gamma: \mathbb{R}^{d} \rightarrow \mathbb{R}$, a column vector $G \in \mathbb{R}^{d \times 1}$, and a continuously differentiable function $\tau: \mathbf{W} \rightarrow \mathbb{R}^{d}$ satisfying

$$
\begin{aligned}
\frac{\partial \tau}{\partial \mathbf{w}} s(\mathbf{w}) & =F(\tau(\mathbf{w}))+G \gamma(\tau(\mathbf{w})) \quad \forall \mathbf{w} \in \mathbf{W} \\
u^{\star}(\mathbf{w}) & =\gamma(\tau(\mathbf{w}))
\end{aligned}
$$

and such that

$$
\operatorname{graph}(\tau)=\left\{(\mathbf{w}, \xi) \in \mathbf{W} \times \mathbb{R}^{d}: \xi=\tau(\mathbf{w})\right\}
$$

is locally asymptotically stable for the system

$$
\dot{\mathbf{w}}=s(\mathbf{w}) \quad \dot{\xi}=F(\xi)+G u^{\star}(\mathbf{w})
$$

with a domain of attraction of the form $\mathbf{W} \times \mathcal{D}^{\prime}$ with $\mathcal{D}^{\prime} \supset \Xi$. As a matter of fact the following result holds (see [7]).

Proposition 1: Let the minimum-phase Assumption 1 hold. Let $(F(\cdot), G, \gamma(\cdot))$ be chosen to satisfy (3) for some $\operatorname{map} \tau(\cdot)$ and so that graph $(\tau)$ is locally asymptotically stable for (4) with domain of attraction $\mathbf{W} \times \mathcal{D}^{\prime}$. Then there exists a continuous function $\kappa: \mathbb{R} \rightarrow \mathbb{R}$ such that the controller

$$
\begin{aligned}
\dot{\xi} & =F(\xi)+G(v+\gamma(\xi)) \\
u & =\gamma(\xi)+v \\
v & =-\kappa(e)
\end{aligned}
$$

solves the problem of nonlinear output regulation with $\Xi \subset$ $\mathcal{D}^{\prime}$.
Remark 1: Under the additional assumptions that the sets $\operatorname{graph}(\pi)$ and $\operatorname{graph}(\tau)$ are also locally exponentially stable for (2) and (4), respectively, and that the function $\gamma(\cdot)$ is locally Lipschitz, the result in the previous proposition holds with $v=-k e$ with $k$ a sufficiently large number.

According to the previous result, the problem of output regulation, for the considered class of systems, reduces to the problem of designing the triplet $(F(\cdot), G, \gamma(\cdot))$ with the required properties. A triplet fulfilling the properties in question is usually said to have the internal model property. It turns out that a number of methodologies to design triplets with the internal model property have been proposed so far. In this respect the following result (proved in [7]) is conceptually relevant as it shows that a triplet with the required properties can be always designed.

Proposition 2: Let $d \geq 2 s+2$. There is an integer $\ell>0$ such that, for almost all choices (see [7] for details) of a controllable pair $(F, G) \in \mathbb{R}^{d \times d} \times \mathbb{R}^{d \times 1}$, with $F$ a Hurwitz matrix whose eigenvalues have real part which is less than $-\ell$, then there exists a continuous function $\gamma: \mathbb{R}^{d} \rightarrow \mathbb{R}$ such that the triplet $(F \xi, G, \gamma(\xi))$ has the internal model property.

Although conceptually relevant, the previous result is weak in terms of practical construction of the regulator as far as the design of the function $\gamma$ is concerned (see [8] for approximated expressions for $\gamma$ of practical interest). Furthermore, it only guarantees the existence of a continuous function $\gamma$.

More constructive design methodologies and more regularity in the controller can be obtained at the price of restricting the class of possible functions $u^{\star}(\mathbf{w})$ entering in the design of the regulator.

Specifically, it is known (see [2]) that the design of the functions in question can be effectively done in the case there exists a locally Lipschitz and bounded map $\phi: \mathbb{R}^{d} \rightarrow \mathbb{R}$ such that

$$
L_{s}^{d} u^{\star}(\mathbf{w})=\phi\left(u^{\star}(\mathbf{w}), L_{s} u^{\star}(\mathbf{w}), \ldots, L_{s}^{d-1} u^{\star}(\mathbf{w})\right)
$$

$\forall \mathbf{w} \in \mathbf{W}$. In fact, in such a case, the choice

$$
F(\xi)=\left(\begin{array}{c}
\xi_{1} \\
\vdots \\
\xi_{d-1} \\
\phi\left(\xi_{0}, \ldots, \xi_{d-1}\right)
\end{array}\right)-G \xi_{0}
$$

with $\xi=\left(\xi_{0}, \ldots, \xi_{d-1}\right)$ makes (3) fulfilled with

$$
\tau(\mathbf{w})=\left(\begin{array}{c}
\tau_{0}(\mathbf{w}) \\
\vdots \\
\tau_{d-1}(\mathbf{w})
\end{array}\right):=\left(\begin{array}{c}
u^{\star}(\mathbf{w}) \\
\vdots \\
L_{s(\mathbf{w})}^{d-1} u^{\star}(\mathbf{w})
\end{array}\right)
$$

and $\gamma(\xi)=\xi_{0}$, for any vector $G$. Furthermore, the theory of high-gain observers (see [5], [12]) can be successfully used in this context to show that if the vector $G$ is chosen as

$$
G=\left(\begin{array}{llll}
g \lambda_{0} & g^{2} \lambda_{1} & \ldots & g^{d} \lambda_{d-1}
\end{array}\right)^{T}
$$


where $\left(\lambda_{0}, \lambda_{1}, \ldots, \lambda_{d-1}\right)$ are coefficients of an Hurwitz polynomial and $g>0$ is an high-gain parameter, then the set $\operatorname{graph}(\tau)$ is locally exponentially stable for the system (4) with a domain of attraction which can be arbitrarily enlarged by taking $g$ sufficiently large.

We summarize the general (constructive) result in the forthcoming proposition that is proved in Appendix. The result is given by considering a general case in which relation (6) is satisfied modulo a residual bias (see (10)) introduced to handle the general theory (internal models with "under-estimated" dimension) presented in Section III.

Proposition 3: Let Assumption 1 be fulfilled. Let $\phi$ : $\mathbb{R}^{d} \rightarrow \mathbb{R}$ be a locally Lipschitz function and $\nu: \mathbf{W} \rightarrow \mathbb{R}$ a bounded function such that

$$
L_{s}^{d} u^{\star}(\mathbf{w})=\phi\left(\tau_{0}(\mathbf{w}), \tau_{1}(\mathbf{w}), \ldots, \tau_{d-1}(\mathbf{w})\right)+\nu(\mathbf{w}) .
$$

Then, there exist a $g^{\star}>0$, a $c>0$, and a continuous function $\kappa: \mathbb{R} \rightarrow \mathbb{R}$ such that for all $g \geq g^{\star}$ the trajectories of the system (1) in closed-loop with the regulator (5), (7), (9) and $\gamma(\xi)=\xi_{0}$ are bounded and such that

$$
\lim _{t \rightarrow \infty} \sup |e(t)| \leq \frac{c}{g^{d+1}} \max _{\mathbf{w} \in \mathbf{W}}|\nu(\mathbf{w})|
$$

It is worth noting that, if (6) holds, the previous result provides an effective way to design asymptotic regulators. On the other hand, in case (10) is satisfied with a nonzero function $\nu(\mathbf{w})$, relation (11) shows the presence of a persistent steady-state regulation error whose amplitude, though, can be arbitrarily decreased by acting on the highgain parameter $g$.

In this work the general theory summarized above will be applied to the relevant case of adaptive output regulation (see [11]). In particular, with $\mathbf{w}=\operatorname{col}(\omega, w)$, we let the exosystem dynamics be given by

$$
\begin{aligned}
& \dot{\omega}=0 \quad \omega \in \Omega \subset \mathbb{R}^{r} \\
& \dot{w}=S(\omega) w \quad w \in W \subset \mathbb{R}^{2 r}
\end{aligned}
$$

where

$S(\omega)=\operatorname{blkdiag}\left(S_{1}\left(\omega_{1}\right), \ldots, S_{r}\left(\omega_{r}\right)\right) \quad S_{i}=\left(\begin{array}{cc}0 & \omega_{i} \\ -\omega_{i} & 0\end{array}\right)$,

and we suppose that the function $u^{\star}(\omega, w)$ introduced above is given by

$$
u^{\star}(\omega, w)=\Gamma(\omega) w \quad \Gamma(\omega)=\left(\begin{array}{lll}
\Gamma_{1}(\omega) & \ldots & \Gamma_{r}(\omega)
\end{array}\right)
$$

with $\Gamma_{i}(\omega) \in \mathbb{R}^{1 \times 2}$ and the pair $(S(\omega), \Gamma(\omega))$ that is assumed, without loss of generality, observable for all $\omega \in \Omega$. The signal $u^{\star}(\mathbf{w}(t))$, in turn, is given by the sum of $r$ harmonics with unknown frequencies $\omega=\left(\omega_{1}, \omega_{2}, \ldots, \omega_{r}\right)$ and unknown amplitudes and phases dependent on the initial condition of $w$. The unknown values of $\omega$ and $w$ are supposed to range on a known compact set $\mathbf{W}=\Omega \times W \subset \mathbb{R}^{r} \times \mathbb{R}^{2 r}$, invariant for (12), better specified in the following.

In the next section a methodology for designing the function $\phi$ fulfilling (6) in the case of exosystems given by
(12) and $u^{\star}$ by (14) will be given. The result relies upon the assumption that the frequencies $\omega_{i}$ are such that $\omega_{i} \neq \omega_{j}$, for all $i, j=1, \ldots, r, i \neq j$, and that $\omega_{i} \neq 0$ for all $i=1, \ldots, r$.

\section{DESIGNING THE ROBUST INTERNAL MODEL}

Within the previous framework of adaptive output regulation, we are interested to develop a design methodology not relying upon the exact knowledge of the number $r$ of harmonics but rather on an estimation $m$, with $m$ not necessarily equal to $r$. We shall design the dimension of the regulator according to the number $m$ of estimated frequencies. If $m$ happens to be $\geq r$, namely the regulator is possibly over-dimensioned with respect to the effective number of exogenous harmonics (in which case "persistence of excitation" conditions are not, in general, guaranteed), asymptotic regulation is achieved. On the other hand, if $m<$ $r$, namely the dimension of the regulator is under-estimated, only practical regulation is obtained with, however, the unavoidable residual regulation error that can be arbitrarily decreased by properly tuning an high-gain parameter of the internal model (in the spirit of Proposition 3).

The state of the regulator is given by the $4 m$ dimensional vector

$$
\xi=\operatorname{col}\left(\xi_{0}, \xi_{1}, \ldots, \xi_{4 m-1}\right)
$$

while its dynamics will be constructed starting from vectors $\ell_{i}(\xi)$, defined as

$$
\ell_{i}(\xi)=\left(\xi_{i} \cdots \xi_{i+2 m-1}\right)^{T}, \quad i=0, \ldots, 2 m,
$$

and matrices $A_{i}(\xi) \in \mathbb{R}^{2 i \times 2 i}, i=1, \ldots, m$, obtained by extracting the first $2 i$ rows and $2 i$ columns from the matrix $A(\xi)$ defined as

$$
A(\xi)=\left(\begin{array}{llll}
\ell_{0}(\xi) & \ell_{1}(\xi) & \cdots & \ell_{2 m-1}(\xi)
\end{array}\right) .
$$

Note that $A_{m}(\xi)=A(\xi)$.

The definition of the matrices $A_{i}$ is instrumental to better characterize the compact set $\mathbf{W}$ in which the state of the exosystem (12) is supposed to range. Specifically, with $\tau(\mathbf{w})$ defined as in (8) with $d=4 m$, namely

$$
\tau(\mathbf{w})=\left(\begin{array}{llll}
u^{\star}(\mathbf{w}) & L_{S} u^{\star}(\mathbf{w}) & \ldots & L_{S}^{4 m-1} u^{\star}(\mathbf{w})
\end{array}\right)^{T}
$$

where $S=S(\omega)$ and $u^{\star}=\Gamma(\omega) w$, the forthcoming regulator design procedure relies upon the assumption that the set $\mathbf{W}$ is invariant for (12) and such that

$$
\left|\operatorname{det}\left(A_{\min \{m, r\}}(\tau(\mathbf{w}))\right)\right| \geq \epsilon \quad \forall \mathbf{w} \in \mathbf{W}
$$

for some $\epsilon>0$. Comments about the previous condition are postponed after the forthcoming proposition.

The value of $\epsilon$ is used in the design of the regulator. Specifically, we let $A_{i \text {, sat }}(\xi)^{-1}$, for $i=1, \ldots, m$, be any (at least locally Lipschitz) bounded matrix that agrees with $A_{i}(\xi)^{-1}$ for all $\xi$ such that $\left|\operatorname{det} A_{i}(\xi)\right| \geq \epsilon$ and $b_{i}(\xi)$ any (at least locally Lipschitz) function satisfying

$$
b_{i}(\xi)= \begin{cases}1 & \text { if }\left|\operatorname{det} A_{i}(\xi)\right| \geq \epsilon \\ 0 & \text { if }\left|\operatorname{det} A_{i}(\xi)\right| \leq \frac{\epsilon}{2}\end{cases}
$$


With the previous notations in hand, we are in the position of formulating the next result that, in conjunction with Proposition 3, yields an adaptive regulator.

Proposition 4: Let

$$
\phi(\xi)=\sum_{i=1}^{m} \alpha_{i}(\xi) \phi_{i}(\xi)
$$

where, for $i=1, \ldots, m$, the $\phi_{i}$ 's are defined as ${ }^{1}$

$$
\phi_{i}(\xi)=\left\lfloor\ell_{2 m}(\xi)\right\rfloor_{i}^{T} A_{i, \mathrm{sat}}(\xi)^{-1}\left\lfloor\ell_{2 m}(\xi)\right\rfloor_{i},
$$

and the $\alpha_{i}$ 's are recursively defined as

$$
\begin{aligned}
\alpha_{m}(\xi) & =b_{m}(\xi) \\
\alpha_{i}(\xi) & =b_{i}(\xi) \prod_{j=i+1}^{m}\left(1-\alpha_{j}(\xi)\right) \quad i=1, \ldots, m-1
\end{aligned}
$$

There exists a bounded function $\nu: \mathbf{W} \rightarrow \mathbb{R}$, with the property that $\nu \equiv 0$ if $m \geq r$, such that relation (10) holds with $d=4 m$ for all $\mathbf{w} \in \mathbf{W}$.

Proof: Let

$$
p_{r}(\lambda)=\lambda^{2 r}+a_{2 r-1} \lambda^{2 r-1}+\ldots+a_{1} \lambda+a_{0}
$$

be the characteristic polynomial of the block-diagonal matrix $S$. By the Cayley-Hamilton theorem, it turns out that $p_{r}(S(\omega))=0$ and thus

$$
\Gamma(\omega) S^{k}(\omega) p_{r}(S(\omega)) w=0
$$

for any $(\omega, w) \in \mathbf{W}$ and any $k \geq 0$. If $r>m$ we introduce the coefficients $c_{i}=a_{i} / a_{2 m}, i=0, \ldots, 2 r-1$, and $c_{2 r}=$ $1 / a_{2 m}$, which are well defined as the coefficient $a_{2 m} \neq 0$ (as all "even" coefficients of the characteristic polynomial of a set of oscillators), and we note that relation (16) implies that

$$
\begin{aligned}
L_{S}^{k+2 m} u^{\star}= & -c_{0} L_{S}^{k} u^{\star}-\ldots-c_{2 m-1} L_{S}^{k+2 m-1} u^{\star} \\
& -c_{2 m+1} L_{S}^{k+2 m+1} u^{\star}-\ldots-c_{2 r} L_{S}^{k+2 r} u^{\star}
\end{aligned}
$$

for all $k \geq 0$, with $S=S(\omega)$ and $u^{\star}=\Gamma(\omega) w$. On the other hand, if $r \leq m$, we let $c_{i}=0, i=0, \ldots, 2(m-r)-1$, and $c_{i}=a_{i-2(m-r)}, i=2(m-r), \ldots, 2 m-1$, and we note that relation (16) implies that

$$
L_{S}^{k+2 m} u^{\star}=-c_{0} L_{S}^{k} u^{\star}-\ldots-c_{2 m-1} L_{S}^{k+2 m-1} u^{\star} .
$$

By collecting the $2 m$ relations obtained by evaluating (17) and (18) for $k=0, \ldots, 2 m-1$, one obtains

$$
\ell_{2 m}(\tau(\mathbf{w}))=-A(\tau(\mathbf{w})) c+Q(\mathbf{w})
$$

where $c=\left(c_{0}, \ldots, c_{2 m-1}\right)^{T}$ and

$Q(\mathbf{w})=-\left(\begin{array}{ccc}L_{S}^{2 m+1} u^{\star} & \ldots & L_{S}^{2 r} u^{\star} \\ \vdots & \ddots & \vdots \\ L_{S}^{4 m} u^{\star} & \ldots & L_{S}^{2 m+2 r-1} u^{\star}\end{array}\right)\left(\begin{array}{c}c_{2 m+1} \\ \vdots \\ c_{2 r}\end{array}\right)$

\footnotetext{
${ }^{1}$ We use the notation $\lfloor v\rfloor_{j}$ to denote a vector in $\mathbb{R}^{2 j}$ obtained by extracting the first $2 j$ components from the vector $v \in \mathbb{R}^{2 d}, d \geq j$.
}

if $r>m$, while $Q(\mathbf{w})=0$ otherwise. Furthermore, relations (17) and (18) evaluated for $k=2 m$ yield

$$
L_{S}^{4 m} u^{\star}(\omega, w)=-\ell_{2 m}(\tau(\mathbf{w}))^{T} c+q(\mathbf{w})
$$

where

$$
q(\mathbf{w})=c_{2 m+1} L_{S}^{4 m+1} u^{\star}-\ldots-c_{2 r} L_{S}^{2(m+r)} u^{\star}
$$

if $r>m$, while $q(\mathbf{w})=0$ otherwise.

We observe that relation (19) implies that the vector $\ell_{2 m}(\tau(\mathbf{w}))-Q(\mathbf{w})$ is in the image of the matrix $A(\tau(\mathbf{w}))$. Furthermore, note that $\operatorname{det}(A(\tau(\mathbf{w})))$ coincides with the Wronskian of the functions $u^{\star}(t), \ldots, u^{\star(2 m-1)}(t)$. For $i=1, \ldots, m$ let $\mathcal{O}_{i} \in \mathbb{R}^{2 i \times 2 r}$ and $\mathcal{C}_{i} \in \mathbb{R}^{2 r \times 2 i}$ be defined as

$$
\begin{aligned}
\mathcal{O}_{i}(\omega) & =\left(\begin{array}{llll}
\Gamma^{T} & S^{T} \Gamma^{T} & \ldots & \left(S^{2 i-1}\right)^{T} \Gamma^{T}
\end{array}\right)^{T} \\
\mathcal{C}_{i}(\omega, w) & =\left(\begin{array}{llll}
w & S w & \ldots & S^{2 i-1} w
\end{array}\right) .
\end{aligned}
$$

It turns out that

$$
A_{i}(\tau(\mathbf{w}))=\mathcal{O}_{i}(\omega) \mathcal{C}_{i}(\omega, w) \quad i=1, \ldots, m .
$$

From this and by the fact that the pair $(S(\omega), \Gamma(\omega))$ is observable for all $\omega \in \Omega$ (which implies that $\operatorname{rank} \mathcal{O}_{i}(\omega)=$ $2 \min \{i, r\}$ for all $i=1, \ldots, m)$, it follows that if $m>r$ then $\operatorname{det}\left(A_{i}(\tau(\mathbf{w}))\right)=0$ for all $i=r+1, \ldots, m$ (as $\left.\operatorname{rank}\left(A_{i}(\tau(\mathbf{w}))\right) \leq 2 r<2 i\right)$, and that $\left|\operatorname{det}\left(A_{r}(\tau(\mathbf{w}))\right)\right| \geq \epsilon$ for all $\mathbf{w} \in \mathbf{W}$ (by (15)). On the other hand, if $m \leq r$, relation (15) implies that $\left|\operatorname{det}\left(A_{m}(\tau(\mathbf{w}))\right)\right| \geq \epsilon$ for all $\mathbf{w} \in \mathbf{W}$. Hence, by letting $i^{\star}=\min \{m, r\}$, it turns out that $\operatorname{rank} A(\tau(\mathbf{w}))=2 i^{\star}$ for all $\mathbf{w} \in \mathbf{W}$ with the first $2 i^{\star}$ columns of $A(\tau(\mathbf{w}))$ that are linearly independent.

Now consider (19) that, for all fixed $\mathbf{w}$ in $\mathbf{W}$, is regarded as a set of $2 m$ linear equations in the unknown $c$. Any solution of (19) can be written as $c=c_{\star}+c_{k}$ where $c_{\star}$ is a solution of (19) and $c_{k} \in \operatorname{Ker} A(\tau(\mathbf{w}))$. By the previous facts it turns out that a possible solution of (19) is given by $c_{\star}=\operatorname{col}\left(c_{\star}^{\prime}, 0\right)$ with

$$
c_{\star}^{\prime}=-A_{i^{\star}}(\tau(\mathbf{w}))^{-1}\left\lfloor\ell_{2 m}(\tau(\mathbf{w}))-Q(\mathbf{w})\right\rfloor_{i^{\star}} .
$$

Hence, using $c=c_{\star}+c_{k}$ and $c_{k} \in \operatorname{Ker} A(\tau(\mathbf{w}))$ in (20), we have

$$
\begin{aligned}
& L_{S}^{4 m} u^{\star}(\mathbf{w})=q(\mathbf{w})-\ell_{2 m}(\tau(\mathbf{w}))^{T} \\
& {\left[\left(\begin{array}{c}
-A_{i^{\star}}(\tau(\mathbf{w}))^{-1}\left\lfloor\ell_{2 m}(\tau(\mathbf{w}))-Q(\mathbf{w})\right\rfloor_{i^{\star}} \\
0
\end{array}\right)+c_{k}\right]}
\end{aligned}
$$

namely, using (19), the fact that $A(\tau(\mathbf{w}))=A(\tau(\mathbf{w}))^{T}, c_{k} \in$ $\operatorname{Ker} A(\tau(\mathbf{w}))$, and that $Q^{T} c_{k}=0($ as $\operatorname{Ker} A(\tau(\mathbf{w}))=\{0\}$ whenever $Q \neq 0$ )

$$
\begin{aligned}
L_{S(\omega)}^{4 m} u^{\star}(w)=\left\lfloor\ell_{2 m}(\tau(\mathbf{w}))\right\rfloor_{i^{\star}}^{T} A_{i^{\star}}(\tau(\mathbf{w}))^{-1} . & \\
& \left\lfloor\ell_{2 m}(\tau(\mathbf{w}))\right\rfloor_{i^{\star}}+\nu(\mathbf{w})
\end{aligned}
$$

where

$\nu(\mathbf{w})=-\left\lfloor\ell_{2 m}(\tau(\mathbf{w}))\right\rfloor_{i^{\star}}^{T} A_{i^{\star}}(\tau(\mathbf{w}))^{-1}\lfloor Q(\mathbf{w})\rfloor_{i^{\star}}+q(\mathbf{w})$.

Using the fact that $\operatorname{det} A_{i^{\star}}(\tau(\mathbf{w})) \geq \epsilon$ (and thus $\left.A_{i^{\star}, \text { sat }}(\tau(\mathbf{w}))^{-1}=A_{i^{\star}}(\tau(\mathbf{w}))^{-1}\right)$, it turns out that

$\phi_{i^{\star}}(\tau(\mathbf{w}))=\left\lfloor\ell_{2 m}(\tau(\mathbf{w}))\right\rfloor_{i^{\star}}^{T} A_{i^{\star}}(\tau(\mathbf{w}))^{-1}\left\lfloor\ell_{2 m}(\tau(\mathbf{w}))\right\rfloor_{i^{\star}}$. 
From this, the definition of $\phi$ and the fact that, by the definition of the $\alpha_{i}$ 's, $\alpha_{i^{\star}}(\tau(\mathbf{w}))=1$ and $\alpha_{i}(\tau(\mathbf{w}))=0$ for all $i \neq i^{\star}$, the result of Proposition 4 follows.

We conclude the section with a few words to comment the requirement (15) and the consequent definition of $\mathbf{W}$. If $m$ happens to be $\geq r$, the requirement in question is fulfilled provided that the state of the exosystem ranges in a compact set $\Omega \times W$, with $\Omega$ a compact set of $\mathbb{R}^{r}$, and $W$ of the form

$$
W=W_{\underline{a}, \bar{a}} \times \cdots \times W_{\underline{a}, \bar{a}} \in \mathbb{R}^{2 r}
$$

with $W_{\underline{a}, \bar{a}}=\left\{w \in \mathbb{R}^{2}:|w| \in[\underline{a}, \bar{a}]\right\}$ for some positive $\underline{a} \leq \bar{a}$. As a matter of fact, note that

$$
A_{r}(\tau(\mathbf{w}))=\mathcal{O}_{r}(\omega) \mathcal{C}_{r}(\omega, w)
$$

with $\mathcal{O}_{r} \in \mathbb{R}^{2 r \times 2 r}$ and $\mathcal{C}_{r} \in \mathbb{R}^{2 r \times 2 r}$ defined in the proof of the previous proposition. The latter matrices are indeed non singular if $(S(\omega), \Gamma(\omega))$ is observable for all $\omega \in \Omega$ and if $W$ has the structure indicated in (21). Hence (15) holds for some $\epsilon>0$ dependent on $\underline{a}$ and $\Omega$. On the other hand, if $m<r$, condition (15) asks that the Wronskian of the functions $u^{\star}(t), \ldots, u^{\star(2 m-1)}$, given by $\operatorname{det} A_{m}(\tau(\mathbf{w}))$, is bounded away from zero. In other words it is required that, among the $r$ harmonics characterizing the signal $u^{\star}(\mathbf{w}(t))$, there exist at most $m$ "dominant" components whose amplitudes are large if compared with the ones of the remaining $r-m$. The next proposition clarifies this aspect by better characterizing a possible allowed set $\mathbf{W}$.

Proposition 5: Let $r>m$. Let $\underline{a} \leq \bar{a}$ be fixed positive numbers and $\Omega$ a fixed compact set of $\mathbb{R}^{r}$. Then there exists a $\bar{\sigma}>0$ such that for all positive $\sigma \leq \bar{\sigma}$ the set $\mathbf{W}=\Omega \times W$, with $W$ of the form

$$
W=\underbrace{W_{\underline{a}, \bar{a}} \times \cdots \times W_{\underline{a}, \bar{a}}}_{m \text { times }} \times \underbrace{W_{\sigma \underline{a}, \sigma \bar{a}} \times \cdots \times W_{\sigma \underline{a}, \sigma \bar{a}}}_{r-m \text { times }},
$$

is invariant for (12) and satisfies $\left|\operatorname{det}\left(A_{m}(\tau(\mathbf{w}))\right)\right| \geq \epsilon$ for some $\epsilon>0$.

\section{PRELIMINARY RESUlTS ABOUT NONLINEAR BUT LINEARLY PARAMETERIZED UNCERTAIN EXOSYSTEMS}

The proposed method can be extended also to the case of exosystems which are nonlinear but linear in the uncertain parameters. As illustrative example, we consider the case in which the function $u^{\star}(t)$ is generated by a van der Pol oscillator and thus satisfies the differential equation

$$
\ddot{u}^{\star}+\omega_{0}^{2} u^{\star}=\epsilon \dot{u}^{\star}-\frac{\epsilon}{3}\left[\dot{u}^{\star}\right]^{3}
$$

where $\omega:=\left(\omega_{0}^{2}, \epsilon\right)^{T}$ are uncertain constant parameters taking values in a compact set $\Omega \subset \mathbb{R}^{2}$. The exosystem $\dot{\mathbf{w}}=s(\mathbf{w})$ can be written as $\dot{\omega}=0$ and

$$
\dot{w}_{0}=w_{1} \quad \dot{w}_{1}=\epsilon w_{1}-\frac{\epsilon}{3} w_{1}^{3}-\omega_{0}^{2} w_{0}
$$

with $u^{\star}=w_{0}$, where $w \in W$ with $W \subset \mathbb{R}^{2}$ the locus where the van der Pol limit cycle takes place. By differentiating once and twice (22), the resulting expressions and (22) can be written as

$$
\left(\begin{array}{c}
\ddot{u}^{\star} \\
u^{\star(3)} \\
u^{\star(4)}
\end{array}\right)=-\left(\begin{array}{cc}
u^{\star} & \frac{1}{3}\left[\dot{u}^{\star}\right]^{3}-\dot{u}^{\star} \\
\dot{u}^{\star} & {\left[\dot{u}^{\star}\right]^{2} \ddot{u}^{\star}-\ddot{u}^{\star}} \\
\ddot{u}^{\star} & 2 \dot{u}^{\star}\left[\ddot{u}^{\star}\right]^{2}+u^{\star(3)}\left(\left[\dot{u}^{\star}\right]^{2}-1\right)
\end{array}\right) \omega
$$

By letting $\xi=\left(\xi_{0}, \xi_{1}, \xi_{2}, \xi_{3}, \xi_{4}\right)^{T}, A(\xi) \stackrel{=}{=}$ $\left(\begin{array}{ll}a_{1}(\xi) & a_{2}(\xi)\end{array}\right)$ with $a_{1}(\xi)=\left(\xi_{0}, \xi_{1}, \xi_{2}\right)^{T}$ and $a_{2}(\xi)=\left(\frac{1}{3} \xi_{1}^{3}-\xi_{1}, \xi_{1}^{2} \xi_{2}-\xi_{2}, 2 \xi_{1} \xi_{2}^{2}+\xi_{3}\left(\xi_{1}^{2}-1\right)\right)^{T}$, $\ell_{1}(\xi)=\left(\begin{array}{ccc}\xi_{2} & \xi_{3} & \xi_{4}\end{array}\right)^{T}$, and $\tau(\mathbf{w})$ the following vector

$$
\left(\begin{array}{c}
\tau_{0}(\mathbf{w}) \\
\tau_{1}(\mathbf{w}) \\
\tau_{2}(\mathbf{w}) \\
\tau_{3}(\mathbf{w}) \\
\tau_{4}(\mathbf{w})
\end{array}\right)=\left(\begin{array}{c}
w_{0} \\
w_{1} \\
-\omega_{0}^{2} w_{0}-\epsilon\left(\frac{1}{3} w_{1}^{3}-w_{1}\right) \\
-\omega_{0}^{2} w_{1}-\epsilon\left(w_{1}^{2}-w_{1}\right) \tau_{2}(\mathbf{w}) \\
-\omega_{0}^{2} \tau_{2}(\mathbf{w})-\epsilon\left(2 w_{1}-1\right) \tau_{2}(\mathbf{w})^{2}- \\
\epsilon\left(w_{1}^{2}-w_{1}\right) \tau_{3}(\mathbf{w})
\end{array}\right)
$$

relation (23) can be compactly rewritten as

$$
\ell_{1}(\tau(\mathbf{w}))=A(\tau(\mathbf{w})) \omega
$$

It turns out that if the matrix $A(\tau(\mathbf{w})) \in \mathbb{R}^{4 \times 2}$ has rank 2 for all $\mathbf{w} \in \Omega \times W$, and thus it is left invertible, relation (24) implies

$$
\omega=\left(A(\tau(\mathbf{w}))^{T} A(\tau(\mathbf{w}))\right)^{-1} A(\tau(\mathbf{w}))^{T} \ell_{1}(\tau(\mathbf{w})) .
$$

Now, let $\ell_{2}(\xi)^{T}:=\left(\begin{array}{cc}\xi_{3} & 2 \xi_{2}^{3}+6 \xi_{1} \xi_{2} \xi_{3}+\xi_{4}\left(\xi_{1}^{2}-1\right)\end{array}\right)$. A simple computation show that, by differentiating four times relation (22) and using (25),

$$
\begin{array}{r}
L_{s} \tau_{4}(\mathbf{w})=\quad \ell_{2}(\tau(\mathbf{w}))^{T}\left(A(\tau(\mathbf{w}))^{T} A(\tau(\mathbf{w}))\right)^{-1} \\
\cdot A(\tau(\mathbf{w}))^{T} \ell_{1}(\tau(\mathbf{w})) .
\end{array}
$$

The previous computations suggest a way to compute a function $\phi$ satisfying (6) and thus to design a regulator. As a matter of fact, let $\varepsilon>0$ defined as

$$
\varepsilon=\min _{\mathbf{w} \in \Omega \times W}\left|\operatorname{det}\left(A(\tau(\mathbf{w}))^{T} A(\tau(\mathbf{w}))\right)\right|
$$

and let $\sigma(\xi)$ be a smooth bounded $2 \times 2$ matrix which agrees with $\left(A(\xi)^{T} A(\xi)\right)^{-1}$ for all $\xi$ such that $\left|\operatorname{det}\left(A(\xi)^{T} A(\xi)\right)\right| \geq$ $\varepsilon$. Then, by (26), the function

$$
\phi(\xi)=\ell_{2}(\xi)^{T} \sigma(\xi) A(\xi)^{T} \ell_{1}(\xi)
$$

is such that $L_{s} \tau_{4}(\mathbf{w})=\phi(\tau(\mathbf{w}))$ by which the result follows.

\section{Conclusions}

This paper presented a new approach to design adaptive internal model-based regulators for a class of minimumphase nonlinear systems. With respect to existing approaches, the proposed method does not rely upon an explicit adaptation method of the control law. The new method has been developed in a general framework handling both the case of over- and under-dimensioned internal models. In the case of under-dimensioned internal models we showed how the proposed controller ensures a bounded steady state regulation error that can be arbitrarily decreased by acting on a design 
parameter. Future works on this subject will be focused on extending the design methodology also to the class of nonlinear but linearly parametrized uncertain exosystems, only marginally addressed in this work, and on numerical validation of the proposed approach and comparison with existing methods.

\section{REFERENCES}

[1] C. Bonivento, A. Isidori, L. Marconi, A. Paoli, "Implicit Fault Tolerant Control: Application to Induction Motors", Automatica, Vol. 40, pp. 355-371, 2004.

[2] C.I. Byrnes and A. Isidori, "Nonlinear Internal Models for Output Regulation", IEEE Trans. on Automatic Control, 49, pp. 2244-2247, 2004.

[3] F. Delli Priscoli, L. Marconi, A. Isidori, "A New Approach to Adaptive Nonlinear Regulation", SIAM Journal on Control and Optimization, 45(3), pp. 829-855, 2006.

[4] Z. Ding, "Global stabilization and disturbance suppression of a class of nonlinear systems with uncertain internal model”, Automatica, 39(3), pp. 471-479, 2003.

[5] F. Esfandiari and H. Khalil, "Output feedback stabilization of fully linearizable systems”, International Journal of Control, 56, pp. 10071037, 1992.

[6] L. Liu, Z. Chen, and J. Huang, "Parameter convergence and minimal internal model with an adaptive output regulation problem" Automatica, 45, 2009, pp. 1206 - 1311, 2009.

[7] L. Marconi, L. Praly, A. Isidori, "Output Stabilization via Nonlinear Luenberger Observers", SIAM Journal on Control and Optimization, Vol. 45, n. 6, pp. 2277-2298, 2007.

[8] L. Marconi, L. Praly, "Uniform Practical Output Regulation", IEEE Transaction on Automatic Control, Vol 53, n. 5, pp. 1184-1202, 2008.

[9] R. Marino, P. Tomei, "Output regulation for linear systems via adaptive internal model”, IEEE Transaction on Automatic Control, 48(12), pp. 2199 - 2202, 2003.

[10] A. Serrani, "Rejection of harmonic disturbances at the controller input via hybrid adaptive external models", Automatica, 42, pp. 1977-1985, 2006.

[11] A. Serrani, A. Isidori and L. Marconi, "Semiglobal nonlinear output regulation with adaptive internal model", IEEE Trans. Autom. Contr, 46(8), pp. 1178-1194, 2001.

[12] A.R. Teel and L. Praly, "Tools for semiglobal stabilization by partial state and output feedback", SIAM Journal on Control Optimization, 33, pp. 1443-1485, 1995.

[13] X . Ye, and J. Huang, "Decentralized adaptive output regulation for a class of large-scale nonlinear systems" IEEE Transactions on Automatic Control, 48, pp. 276-281, 2003

\section{APPENDIX}

\section{A. Proof of Proposition 3}

We consider the change of variable $\xi \rightarrow \chi:=\xi-\tau(\mathbf{w})$, transforming system (1), (5), with $F$ as in (7) and $\gamma(\xi)=\xi_{0}$, as

$$
\begin{aligned}
\dot{\mathbf{w}} & =s(\mathbf{w}) \\
\dot{z} & =f(\mathbf{w}, z, e) \\
\dot{\tilde{\xi}} & =A \tilde{\xi}+B(\tilde{\phi}(\tilde{\xi}, \mathbf{w})-\nu(\mathbf{w}))+G v \\
\dot{e} & =q(\mathbf{w}, z, e)+\tau_{0}(\mathbf{w})+\tilde{\xi}_{0}+v
\end{aligned}
$$

where $A$ is the "shift" matrix given by all zeros except the elements of the superdiagonal which are all ones, $B=$

$$
\begin{aligned}
\left(\begin{array}{llll}
0 & \cdots & 0 & 1
\end{array}\right)^{T}, \\
\tilde{\phi}(\tilde{\xi}, \mathbf{w})=\phi(\tilde{\xi}+\tau(\mathbf{w}))-\phi(\tau(\mathbf{w})) .
\end{aligned}
$$

Note that $\tilde{\phi}(\tilde{\xi}, \mathbf{w})$ is locally Lipschitz and bounded for all $\tilde{\xi} \in$ $\mathbb{R}^{d}$ and $\mathbf{w} \in \mathbf{W}$, and that $\tilde{\phi}(0, \mathbf{w})=0$ for all $\mathbf{w} \in \mathbf{W}$. Note also that $q(\mathbf{w}, z, 0)+\tau_{0}(\mathbf{w})=0$ for all $(\mathbf{w}, z) \in \operatorname{graph}(\pi)$. By the further change of variable (meant to put system (27) in normal form) $\tilde{\xi} \rightarrow \chi:=\tilde{\xi}-G e$, system (27) transforms as

$$
\begin{aligned}
\dot{\mathbf{w}}= & s(\mathbf{w}) \\
\dot{z}= & f(\mathbf{w}, z, e) \\
\dot{\chi}= & A \chi+B(\tilde{\phi}(\chi, \mathbf{w})-\nu(\mathbf{w})) \\
& -G\left(q(\mathbf{w}, z, 0)+\chi_{0}+\tau_{0}(\mathbf{w})\right)+L(\mathbf{w}, z, \chi, e) \\
\dot{e}= & q(\mathbf{w}, z, e)+\tau_{0}(\mathbf{w})+\chi_{0}+g \lambda_{0} e+v
\end{aligned}
$$

where

$$
\begin{aligned}
L=A G e+B & (\tilde{\phi}(\chi+G e, \mathbf{w})-\tilde{\phi}(\chi, \mathbf{w})) \\
& -G\left(q(\mathbf{w}, z, e)-q(\mathbf{w}, z, 0)+g \lambda_{0} e\right) .
\end{aligned}
$$

Note that $L(\mathbf{w}, z, \chi, 0)=0$ for all $(\mathbf{w}, z, \chi) \in \mathbf{W} \times \mathbb{R}^{n} \times \mathbb{R}^{d}$. By following the high-gain observer theory (see [5], [12]), we finally re-scale the $\chi$ variable as

$$
\chi=D_{g} \tilde{\chi} \quad \text { with } \quad D_{g}=\operatorname{diag}\left(1, g, \ldots, g^{d-1}\right)
$$

by thus transforming the $\chi$ and $e$ dynamics in (28) as

$$
\begin{aligned}
\dot{\tilde{\chi}}= & g H \tilde{\chi}+\frac{1}{g^{d-1}} B\left(\tilde{\phi}\left(D_{g} \tilde{\chi}, \mathbf{w}\right)-\nu(\mathbf{w})\right) \\
& -g \Lambda y_{\mathrm{z}}(\mathbf{w}, z)+D_{g}^{-1} L\left(\mathbf{w}, z, D_{g} \tilde{\chi}, e\right) \\
\dot{e}= & q(\mathbf{w}, z, e)+\tau_{0}(\mathbf{w})+\tilde{\chi}_{0}+g \lambda_{0} e+v
\end{aligned}
$$

where $\Lambda=\left(\begin{array}{ccc}\lambda_{0} & \cdots & \lambda_{d-1}\end{array}\right)^{T}, y_{z}=q(\mathbf{w}, z, 0)+\tau_{0}(\mathbf{w})$, and $H$ is a Hurwitz matrix. The overall closed-loop system, regarded as a system with input $v$ and output $e$, has relative degree one and zero dynamics given by

$$
\begin{aligned}
\dot{\mathbf{w}} & =s(\mathbf{w}) \\
\dot{z} & =f(\mathbf{w}, z, 0) \\
\dot{\tilde{\chi}} & =g H \tilde{\chi}+\frac{1}{g^{d-1}} B\left(\tilde{\phi}\left(D_{g} \tilde{\chi}, \mathbf{w}\right)-\nu(\mathbf{w})\right)-g \Lambda y_{\mathrm{z}} .
\end{aligned}
$$

Standard ISS arguments, using the fact that $H$ is Hurwitz and that $\tilde{\phi}$ is a bounded locally Lipschitz function, can be used to show that there exists a $g^{\star}>0$ such that for all $g \geq g^{\star}$ the $\tilde{\chi}$-subsystem, regarded as a system with inputs $\left(y_{\mathrm{z}}, \nu\right)$, is ISS. In particular there exists a positive $c^{\prime}$ such that the following asymptotic estimate holds

$$
\lim _{t \rightarrow \infty} \sup |\tilde{\chi}(t)| \leq c^{\prime} \max \left\{\frac{1}{g^{d}} \lim _{t \rightarrow \infty} \sup |\nu(\mathbf{w}(t))|,\right.
$$

By using the minimum-phase assumption, the fact that $y_{\mathrm{z}}(\mathbf{w}, z)=0$ for all $(\mathbf{w}, z) \in \operatorname{graph}(\pi)$, standard cascade arguments can be used to conclude that also system (29) is ISS with an asymptotic estimate of the form

$$
\begin{aligned}
& \lim _{t \rightarrow \infty} \sup |(\mathbf{w}(t), z(t), \tilde{\chi}(t))|_{\operatorname{graph}(\pi) \times\{0\}} \leq \\
& \frac{c^{\prime}}{g^{d}} \lim _{t \rightarrow \infty} \sup \mid \nu(\mathbf{w}(t) \mid .
\end{aligned}
$$

From this, the fact that $q(\mathbf{w}, z, 0)+\tau_{0}(\mathbf{w})=0$ for all $(\mathbf{w}, z) \in \operatorname{graph}(\pi)$, the small gain arguments of [7] lead to conclude that there exists a continuous $\kappa(\cdot)$ such that the claim of Proposition 4 holds for some positive $c$. Furthermore, the $\kappa(\cdot)$ is linear if $\operatorname{graph}(\pi)$ is also locally exponentially stable for (2). 